\title{
Comparison of Inferior Turbinate Reduction Techniques on Postoperative Epistaxis and Nasal Congestion
}

\author{
Emily S. Sagalow, BS*; Vanessa Christopher, MD'; Kurren Gill, MD'; Raphael Banoub, MD'; \\ Somya Shankar, BS1; Madalyne Sunday, BS'; Tingting Zhan, PhD²; Glen D'Souza, MD; Joel \\ Stanek, MD'; Michelle Hwang, MD; Ryan Heffelfinger, MD'; Howard Krein, MD, PhD
}

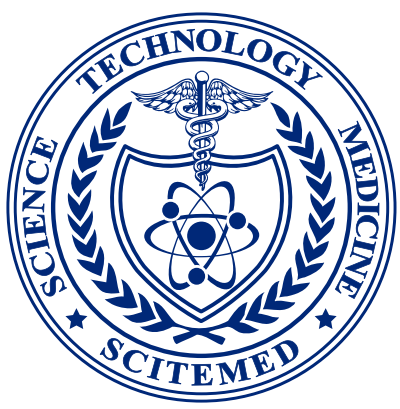

'Department of Otolaryngology-Head and Neck Surgery, Thomas Jefferson University Hospital, Philadelphia, PA, USA

2Division of Biostatistics, Department of Pharmacology and Experimental Therapeutics, Thomas Jefferson University, Philadelphia, PA, USA

\section{ABSTRACT}

Background: Inferior turbinate reduction is often performed concurrently during functional nasal surgery. Surgical techniques include partial excision and submucous resection. Common postoperative complications after nasal surgery include epistaxis and recurrent or persistent congestion.

Objective: The aim of the study was to determine the impact of inferior turbinate reduction technique on postoperative epistaxis and recurrent or persistent nasal congestion.

Methods: Patients who underwent nasal surgery at a single academic institution between January 2017 and December 2020 were identified. Data collected included demographics, risk factors, intraoperative techniques, and postoperative course. Main outcome measures were postoperative epistaxis and nasal congestion.

Results: Of the 219 nasal surgery patients who received concurrent inferior turbinate reduction, 146 (66.7\%) underwent partial excision of the inferior-lateral aspect with a thru-cut and 73 (33.3\%) underwent submucous resection using an ultrasonic bone aspirator (Stryker Sonopet $®$ ). Postoperative epistaxis and recurrent or persistent nasal congestion were not significantly associated with either inferior turbinate reduction technique.

Conclusion: A comparison of two methods of inferior turbinate reduction, partial excision and submucous resection, did not show a difference in risk of postoperative epistaxis or nasal congestion. Both techniques demonstrate safety and utility in inferior turbinate reduction.
\end{abstract}

\section{INTRODUCTION}

Inferior turbinate hypertrophy is commonly found in patients with nasal obstruction and is often concurrent with septal deviation. Medical therapy for inferior turbinate hypertrophy includes an antihistamine or nasal decongestant spray [1]. If hypertrophied inferior turbinates are refractory to medical management, surgical reduction of the turbinates can be performed $[2,3]$.

Several techniques exist for surgical inferior turbinate reduction. Submucous resection of the inferior turbinates involves removing the underlying bone and erectile tissue of the turbinates while sparing the mucosal tissue $[4,5]$. In contrast, partial excision of the inferior turbinates involves trimming portions of the inferior turbinate bone along with its overlying mucosa [4]. Both techniques are often performed in conjunction with outfracturing of the inferior turbinates, which lateralizes the entire turbinate structure and expands the internal nasal valve. Other surgical methods for inferior turbinate reduction include total turbinectomy, microdebrider removal, electrocautery, laser cautery, cryotherapy, and radiofrequency ablation $[1,3-4,6-9]$.

Turbinate reduction surgery can be associated with adverse postoperative outcomes such as postoperative epistaxis, nasal congestion, infection, and nerve injury, and empty nose syndrome $[4,10]$. There is literature which comments on the rate of postoperative complications and the type of surgical technique utilized. For example, previous studies described rates of epistaxis to be $1.6 \%$ after submucous resection with a microdebrider and $5.8 \%$ after radical turbinectomy $[9,11]$. Other studies comment on nasal congestion: $96 \%$ of patients reported an improvement in nasal breathing two weeks after surgery, and $88 \%$ after two months, after undergoing bilateral inferior turbinate reduction. This was compared to $78 \%$ of patients who reported improvement after two weeks, and 76\% after two months, after a submucosal diathermy turbinate reduction [12].

The purpose of this study was to compare two surgical techniques of inferior turbinate reduction, submucous resection versus partial excision, with their associated postoperative complications including epistaxis and recurrent nasal congestion in patients undergoing functional nasal surgery at a single academic center. To date, no other study has directly compared these two methods with endpoints of epistaxis and nasal congestion.

\section{METHODS}

This study was approved by the Thomas Jefferson University Institutional Review Board. Retrospective identification of patients who underwent rhinoplasty with inferior turbinate reduction at a single academic institution between January 2017 and December 2020 was completed. Data collected included demographics, risk factors, intraoperative techniques, and postoperative clinical course. Each patient underwent rhinoplasty with inferior turbinate reduction completed one of two ways: a single surgeon utilized the submucous resection technique, and a second surgeon performed the partial excision technique. Patients were excluded from the study if they did not have a minimum follow-up of two months. This time period was chosen based on literature describing the assessment of nasal breathing after nasal surgery at the two-month mark [12]. Following this timeframe, a post-procedural period of less than two months was deemed inadequate to assess if 


\begin{tabular}{|c|c|c|}
\hline Variable & $\begin{array}{l}\text { Partial excision of the inferior-lateral aspect } \\
\text { with a thru-cut }(n=146)\end{array}$ & $\begin{array}{l}\text { Submucous resection by an ultrasonic bone } \\
\text { aspirator }(n=73)\end{array}$ \\
\hline \multicolumn{3}{|l|}{ Gender } \\
\hline Female, $\mathrm{n}(\%)$ & $89(61)$ & $46(63)$ \\
\hline Male, n (\%) & $57(39)$ & $27(37)$ \\
\hline Average age, yr & $33.5 \pm 13.5$ & $32.0 \pm 13.8$ \\
\hline Average BMI & $24.2 \pm 7.5$ & $23.5 \pm 3.7$ \\
\hline \multicolumn{3}{|l|}{ Smoking status } \\
\hline Never, n (\%) & $113(77.4)$ & $55(75.3)$ \\
\hline Former, n (\%) & $22(15.1)$ & $14(19.2)$ \\
\hline Current, $\mathrm{n}(\%)$ & $11(7.5)$ & $4(5.5)$ \\
\hline History of previous nasal surgery, $n$ (\%) & $26(17.8)$ & $8(11.0)$ \\
\hline History of nasal trauma, n (\%) & $77(52.7)$ & $38(52.1)$ \\
\hline \multicolumn{3}{|l|}{ Rhinoplasty indication } \\
\hline Functional, n (\%) & $100(68.5)$ & $36(49.3)$ \\
\hline Cosmetic, n (\%) & $11(7.5)$ & $14(19.2)$ \\
\hline Both, n (\%) & $35(24.0)$ & $23(31.5)$ \\
\hline Concurrent septoplasty, n (\%) & $109(74.7)$ & $60(82.2)$ \\
\hline
\end{tabular}
BMI, body mass index.

the postoperative complications of interest did or did not occur. Therefore, patients lost to follow up prior to this time were excluded.

\section{Inferior Turbinate Reduction Techniques}

The first technique of inferior turbinate reduction was partial excision of the turbinate (Video 1, available online at https://doi.org/10.24983/scitemed. aohns.2022.00154). In this method, the turbinate is first in-fractured and the inferior-lateral aspect exposed. Starting from just posterior to the head of the turbinate, partial excision is performed with a thru-cut, progressively traveling posteriorly along the length of the turbinate. Hemostasis is achieved with suction bovie cautery, and the turbinate is then outfractured. The second technique is submucous resection of the turbinate, which requires a small incision at the head of the turbinate and elevation of a submucous pocket between the turbinate mucosa and underlying bone with a Cottle freer (Video 2, available online at https://doi.org/10.24983/scitemed.aohns.2022.00154). An ultrasonic bone aspirator (Stryker Sonopet ${ }^{\circledR}$ ) is introduced into the pocket and bone is removed while sparing the turbinate mucosa. Similarly, the turbinate is then outfractured. Cautery is not regularly used with this technique. No postoperative nasal packing was utilized after either technique.

\section{Outcome Measures}

The primary outcome measures were postoperative epistaxis and recurrent or persistent nasal congestion. Epistaxis was defined as notable postoperative bleeding from the nasal cavity either reported by the patient or treated by a clinician. Recurrent or persistent nasal congestion was defined as excessive nasal congestion lasting longer than expected for a patient's postoperative period of two months. The "excessive" nature of the congestion was a subjective assessment reported by the patient. Predictors analyzed included patient age, gender, body mass index (BMI), smoking status, history of previous nasal surgery, history of nasal trauma, concurrent septoplasty, and presence of prolonged nasal crusting or empty nose syndrome. Smoking status was defined as never, former, or current smoker. History of previous nasal surgery included history of turbinate reduction or turbinectomy, functional endoscopic sinus surgery (FESS), rhinoplasty, open reduction internal fixation (ORIF) for nasal fracture, or septoplasty. Prolonged nasal crusting was defined as crusting of the nasal cavity examined via fiber-optic scope lasting longer than expected for the postoperative timeframe as deemed by the surgeon. Empty nose syndrome was defined as a paradoxical subjective complaint of nasal congestion, dryness, or crusting without objective physical evidence of obstruction within the nasal cavity.

\section{Statistical Analysis}

For the statistical analyses, SPSS version 27 software (IBM Corp., Armonk, NY, USA) was used. Univariable analysis was performed on the primary endpoints of epistaxis and recurrent or persistent nasal congestion with predictors using a logistic regression model. Statistical significance was defined as a P-value $<0.05$

\section{RESULTS}

Three hundred and eight patients underwent functional nasal surgery involving inferior turbinate reduction during the selected timeframe. Of those 308 patients, 219 met the two-month postoperative follow-up criteria and were included in the analysis. One hundred and forty-six (66.7\%) underwent inferior turbinate reduction via the partial excision technique, and 73 (33.3\%) underwent inferior turbinate reduction via the submucous resection method. Patient demographics within each group are demonstrated in Table 1. There were no reports of prolonged nasal crusting or empty nose syndrome. The average follow-up period was 12.41 months \pm 11.53 months.

\section{Epistaxis}

For the cohort that underwent partial excision, 7 (4.8\%) patients experienced postoperative epistaxis. For the cohort that received submucous resection, $2(2.7 \%)$ patients experienced postoperative epistaxis ( $P=0.37$ on Fischer's exact test). Never-smokers had a lower probability of epistaxis compared to current smokers (odds ratio $=0.079,95 \%$ confidence interval $=0.010-0.592$, $\mathrm{P}=0.014$ ). All other predictors including patient age, gender, BMl, smoking 


\begin{tabular}{|c|c|c|}
\hline Variable & Odds ratio (95\% Cl) & $\mathrm{P}$ \\
\hline Inferior turbinate reduction technique & & 0.502 \\
\hline Partial excision & $1.841(0.310-10.932)$ & \\
\hline Submucous resection & 1 (reference) & \\
\hline Age at surgery & $0.972(0.908-1.042)$ & 0.422 \\
\hline Gender & & 0.991 \\
\hline Male & $1.019(0.191-5.335)$ & \\
\hline Female & 1 (reference) & \\
\hline BMI & $1.019(0.852-1.218)$ & 0.839 \\
\hline \multicolumn{3}{|l|}{ Smoking status } \\
\hline Former vs Current & $0.099(0.008-1.267)$ & 0.075 \\
\hline Never vs Current & $0.079(0.010-0.592)$ & 0.014 \\
\hline Never vs Former & $1.376(0.08-0.855)$ & 0.998 \\
\hline History of previous nasal surgery & $0.484(0.082-2.854)$ & 0.423 \\
\hline History of nasal trauma & $2.186(0.639-7.479)$ & 0.176 \\
\hline Concurrent Septoplasty & $0.705(0.052-9.493)$ & 0.792 \\
\hline
\end{tabular}

$\mathrm{BMI}$, body mass index; $\mathrm{Cl}$, confidence interval; vs, versus.

status (former vs. current and former vs. never), history of previous nasal surgery, history of nasal trauma, concurrent septoplasty, and performing surgeon were insignificant using univariable logistic regression model and are demonstrated in Table 2. Other risk factors, specifically diabetes (2 out of $219,0.9 \%$ ), coagulation abnormalities ( 1 out of $219,0.45 \%$ ), and the use of anticoagulation or antiplatelet medications ( 8 out of $219,3.65 \%$ ) were not included in this analysis due to the low number of subjects.

\section{Nasal Congestion}

For the cohort that received partial excision, 34 (23.3\%) patients experienced postoperative recurrent or persistent nasal congestion. For the cohort that received submucous resection, 17 (23.3\%) patients experienced postoperative recurrent or persistent nasal congestion $(P=0.57$ using univariable logistic regression). All other predictors including patient age, gender, BMI, smoking status, history of previous nasal surgery, history of nasal trauma, concurrent septoplasty, and performing surgeon were insignificant using univariable logistic regression model and are demonstrated in Table 3. Hence none of the predictors were retained in the multivariable logistic regression model. Other risk factors, specifically diabetes, coagulation abnormalities, and the use of anticoagulation or antiplatelet medications, were not included in this analysis due to the low number of subjects.

\section{DISCUSSION}

Inferior turbinate reduction is commonly performed during rhinoplasty and functional endoscopic sinus surgery to provide symptomatic relief of nasal congestion. There are numerous studies which describe the types of surgical procedures used to perform turbinate reduction and the possible postoperative complications. Established methods used to address turbinate hypertrophy include, but are not limited to, electrocautery, microdebridement, laser, diathermy, and submucosal resection. Currently, there is literature which assesses these techniques and the rates of different postoperative sequelae. For example, Majahan et al. compared the technique of powered turbinectomy with a microdebrider to submucous resection using turbinate scissors and reported decreased epistaxis and persistent nasal obstruction in the submucous resection cohort [13]. Currently, there is limited data comparing two particular techniques of turbinate reduction performed at our institution: partial excision and submucous resection. In the study described in this paper, retrospective review of 219 patients at a single academic center who underwent inferior turbinate reduction demonstrated that neither method was associated with an increased risk of postoperative epistaxis or differences in outcomes of recurrent or persistent nasal congestion.

Bleeding after turbinate reduction is a well-studied complication after nasal surgery. A retrospective review of 359 patients who underwent septoplasty with turbinate reduction cited a $4.5 \%$ rate of postoperative epistaxis [5]. However, the etiology of epistaxis was described as multifactorial and dependent on different variables including concurrent nasal procedures such as septoplasty, method of turbinate reduction, and use/non-use of nasal packing. A separate review reported that the most common postoperative complications for patients who underwent partial excision of the inferior turbinates included crusting (20\% of patients) and rhinorrhea $(11 \%$ of patients) [4]. Another study analyzing 120 patients who underwent submucous resection of the inferior turbinates using a microdebrider found a postoperative bleeding rate of $1.6 \%$ [9]. There is limited data comparing bleeding rates after partial turbinate excision versus submucous turbinate resection, and our results suggest no difference in bleeding outcomes depending on the technique utilized.

Current smokers had a significantly higher amount of epistaxis episodes as a cohort compared to never-smokers in our study $(P=0.014)$. There is a known association between smoking and increased risk of postoperative bleeding after otolaryngologic surgery. An explanation for the relationship between tobacco smoke and postoperative bleeding is tobacco smoke acting as a chemical irritant, and toxins within the smoke irritating fragile vessels prone to rupture which leads to bleeding. A prospective cohort study by Langsted et al. discusses how smoking is associated with increased risk of major bleeding specifically airway, urinary, gastrointestinal and intracranial bleeding. The presence of smoking and intensity of smoking increased the risk of major bleeding, which supports our finding of current smokers experiencing significantly more episodes of epistaxis than never-smokers [14-16].

Recurrent or persistent nasal congestion after turbinate reduction is 


\begin{tabular}{|c|c|c|}
\hline Variable & Odds ratio (95\% Cl) & $\mathrm{P}$ \\
\hline Inferior turbinate reduction technique & & 0.677 \\
\hline Partial excision & $0.858(0.417-1.765)$ & \\
\hline Submucous resection & 1 (reference) & \\
\hline Age at surgery & $1.014(0.986-1.042)$ & 0.326 \\
\hline Gender & & 0.392 \\
\hline Male & $0.755(0.356-2.350)$ & \\
\hline Female & 1 (reference) & \\
\hline BMI & $1.005(0.943-1.600)$ & 0.969 \\
\hline \multicolumn{3}{|l|}{ Smoking status } \\
\hline Former vs Current & $1.034(0.260-4.105)$ & 0.963 \\
\hline Never vs Current & $1.466(0.325-6.601)$ & 0.619 \\
\hline Never vs Former & $0.622(0.297-1.303)$ & 0.208 \\
\hline History of previous nasal surgery & $0.980(0.391-2.461)$ & 0.966 \\
\hline History of nasal trauma & $1.171(0.576-2.379)$ & 0.663 \\
\hline Concurrent Septoplasty & $0.725(0.272-1.934)$ & 0.521 \\
\hline
\end{tabular}

$\mathrm{BMI}$, body mass index; $\mathrm{Cl}$, confidence interval; vs, versus.

another well-established sequelae of nasal surgery, particularly turbinate reduction. Neumann et al. conducted a study which surveyed patients' nasal aeration, sense of smell, rhinorrhea, and sense of smell after septoplasty and turbinate reduction. Preoperatively, $93 \%$ of patients rated their nasal obstruction as moderate (37.23\%) or severe (56.2\%). Postoperatively, $35.29 \%$ of patients still reported a small degree of nasal breathing obstruction [17]. Gupta et al. found that patients rated the severity of daytime nasal obstruction as 2.3 out of 6 after resection of the inferior and lateral aspects of the inferior turbinate using a microdebrider [18]. Other studies reported similar changes in nasal airway obstruction over various timeframes [19]. Our results demonstrated a similar percentage of postoperative nasal obstruction: $17.2 \%$ of patients total, $17.4 \%$ for the partial resection technique and $16.8 \%$ for the submucous resection technique, experienced postoperative recurrent or persistent nasal congestion. A study by $\mathrm{Hol}$ et al. discussed how certain turbinate reduction techniques including turbinectomy and partial resection via cutting and/or trimming were less successful than submucous techniques due to the altered turbinate mucosa [20]. This particular paper suggested complete or partial removal of turbinate mucosa can cause persistent nasal congestion due to 'renewed hyperplasia' of the mucosa and be associated with decreased success in relieving obstruction over time. Interestingly, this contrasts with our findings in that there was no difference in rate of postoperative nasal congestion between the partial excision (mucosal-sacrificing) and submucous resection with Sonopet (mucosal-preserving) techniques.

The rationale for conducting this study was to compare two methods of minimally invasive inferior turbinate reduction methods used at our institution using the outcome measures of postoperative epistaxis and nasal congestion. A review of current literature did not reveal established studies comparing these two techniques and the outcomes of interest. Additionally, it is worth highlighting that several of the aforementioned studies included nasal packing in their cohorts, which differs from our study design, and could affect rates of postoperative epistaxis and congestion [13,5]. Our rates of epistaxis and nasal congestion after turbinate reduction were similar to what has been described in the literature. However, our results are unique in that they show no difference in postoperative complication rate between two surgical techniques which have not previously been compared. This supports the novel nature and utility of this study.

\section{Limitations}

The main limitations of this study are the retrospective nature of data collection and the subjectivity of perceived nasal congestion. Retrospective data collection through patient medical charts allows for outcomes such as epistaxis and recurrent or persistent nasal congestion to be recorded only when mentioned by the patient and documented by a healthcare professional. It is possible there were other episodes of recurrent nasal congestion extending past the follow-up period average of 12.41 months \pm 11.53 months that were not reported by the patient. Longer follow-up would allow for detection of persistent or recurrent nasal congestion as well as delayed presentations of epistaxis. Another confounding factor is the additional procedures often performed in conjunction with turbinate reduction to address functional aspects of nasal congestion. Turbinate reduction as an isolated procedure was rare among our patient population, therefore epistaxis and nasal congestion as postoperative complications cannot be solely attributed to the turbinate reduction procedure. Finally, as a single center study the derived results may be less generalizable despite the large sample size.

\section{Future Directions}

In the future, it would be beneficial to investigate these endpoints in a prospective nature with a defined follow-up period. This would allow for a more definitive and informed evaluation of recurrent nasal congestion. With a prospective study design, rhinomanometry, a functional test of nasal aerodynamics that measures transnasal airflow and pressure gradient, could be completed after inferior turbinate reduction for quantitative analysis and comparison of the two techniques [21].

\section{CONCLUSION}

The goal of this study was to evaluate postoperative complications that can occur after two techniques of inferior turbinate reduction, i.e., partial excision of the inferior-lateral aspect with a thru-cut and submucous resection with an ultrasonic bone aspirator (Stryker Sonopet ${ }^{\circledR}$ ). Our study found no statistical difference between the two techniques in the rate of postoperative epistaxis or recurrent nasal obstruction. Never-smokers had a decreased rate of epistaxis overall. These findings demonstrate that both techniques have reasonable clinical utility with low rates of postoperative epistaxis. 


\section{ARTICLE INFORMATION}

*Correspondence: Emily S. Sagalow, BS, Department of Otolaryngology-Head and Neck Surgery, Thomas Jefferson University Hospital, 925 Chestnut Street, Floor 6, Philadelphia, PA 19107, USA. Email: Emily.Sagalow@jefferson.edu

Received: Oct. 10, 2021; Accepted: Dec. 14, 2021; Published:Jan. 14, 2022

DOI: 10.24983/scitemed.aohns.2022.00154

Author Contributions: Emily S. Sagalow contributed to the design, data collection, analysis, writing/editing of the manuscript; Vanessa Christopher contributed to the writing/editing of the manuscript; Kurren Gill contributed to the design, writing/editing of the manuscript; Raphael Banoub contributed to the data collection of the manuscript; Somya Shankar contributed to the data collection of the manuscript; Madalyne Sunday contributed to the data collection of the manuscript; Tingting Zhan contributed to the analysis of the manuscript; Glen D'Souza contributed to the analysis and the writing/editing of the manuscript; Joel Stanek contributed to the design and the writing/editing of the manuscript; Michelle Hwang contributed to the writing/editing of the manuscript; Ryan Heffelfinger contributed to the design and the writing/editing of the manuscript; Howard Krein contributed to the design and the writing/editing of the manuscript.

Ethics Approval and Consent to Participate: The study is in accordance with the ethical standards of the 1964 Helsinki declaration and its later amendments or comparable ethical standards.

Funding: This research received no specific grant from any funding agency in the public, commercial, or not-for-profit sectors.

Conflict of Interest: The authors report no financial or other conflict of interest relevant to this article, which is the intellectual property of the authors.

Copyright $\odot 2022$ The Author(s). This is an open-access article distributed under the terms of the Creative Commons Attribution 4.0 International License (CC-BY). In accordance with accepted academic practice, anyone may use, distribute, or reproduce this material, so long as the original author(s), the copyright holder(s), and the original publication of this journal are credited, and this publication is cited as the original. To the extent permitted by these terms and conditions of license, this material may not be compiled, distributed, or reproduced in any manner that is inconsistent with those terms and conditions

Publisher Disclaimer: It is pertinent to note that all opinions and statements made by the author(s) throughout this article are solely those of the author(s) They may not be representative of those of their affiliated organizations, the publishing house, editors, or other reviewers since they are the opinions and statements of the author(s) alone. The publisher does not guarantee or endorse any statements made by the manufacturer of any product mentioned in this article or the author's evaluation.

\section{REFERENCES}

1. Rao SUP, Basavaraj P, Yempalle SB, Ramachandra AD. A prospective study of different methods of inferior turbinate reduction. J Clin Diagn Res 2017;11(5):MC01-MC03.
2. Han JK, Stringer SP, Rosenfeld RM, et al. Clinical consensus statement: Septoplasty with or without inferior turbinate reduction. Otolaryngol Head Neck Surg 2015;153(5):708-720.

3. Bergmark RW, Gray ST. Surgical management of turbinate hypertrophy. Otolaryngol Clin North Am 2018;51(5):919-928.

4. Sinno S, Mehta K, Lee ZH, Kidwai S, Saadeh PB, Lee MR. Inferior turbinate hypertrophy in rhinoplasty: Systematic review of surgical techniques. Plast Reconstr Surg 2016;138(3):419e-429e.

5. Joshi RR, Riley CA, Kacker A. Complication rates following septoplasty with inferior turbinate reduction. Ochsner J 2019;19(4):353-356.

6. Mabry RL. Surgery of the inferior turbinates: How much and when? Otolaryngol Head Neck Surg 1984;92(5):571-576.

7. Anderson M, Liang J. Trends in inferior turbinate surgery: Analysis of patients using the Medicare database. Int Forum Allergy Rhinol 2018;8(10):1169-1174.

8. Bhandarkar ND, Smith TL. Outcomes of surgery for inferior turbinate hypertrophy. Curr Opin Otolaryngol Head Neck Surg 2010;18(1):49-53.

9. Friedman M, Tanyeri H, Lim J, Landsberg R, Caldarelli D. A safe, alternative technique for inferior turbinate reduction. Laryngoscope 1999;109(11):1834-1837.

10. Coste A, Dessi P, Serrano E. Empty nose syndrome. Eur Ann Otorhinolaryngol Head Neck Dis 2012;129(2):93-97.

11. Garth RJ, Cox HJ, Thomas MR. Haemorrhage as a complication of inferior turbinectomy: A comparison of anterior and radical trimming. Clin Otolaryngol Allied Sci 1995;20(3):236-238

12. Fradis M, Golz A, Danino J, et al. Inferior turbinectomy versus submucosal diathermy for inferior turbinate hypertrophy. Ann Otol Rhinol Laryngo/ 2000;109(11):1040 1045.

13. Mahajan R, Nagaraj TM, Prashanth V. A prospective study to compare the efficacy of powered turbinectomy versus submucous resection in the surgical management of inferior turbinate hypertrophy. Indian J Otolaryngol Head Neck Surg 2019;71(Suppl 3):2203-2209.

14. Demars SM, Harsha WJ, Crawford JV. The effects of smoking on the rate of postoperative hemorrhage after tonsillectomy and uvulopalatopharyngoplasty. Arch Otolaryngol Head Neck Surg 2008;134(8):811-814.

15. Wang CY, Dudzinski J, Nguyen D, Armbrecht E, Maher IA. Association of smoking and other factors with the outcome of Mohs reconstruction using flaps or grafts. JAMA Facial Plast Surg 2019;21(5):407-413.

16. Langsted A, Nordestgaard BG. Smoking is associated with increased risk of major bleeding: A prospective cohort study. Thromb Haemost 2019;119(1):39-47.

17. Neumann A, Lehmann N, Stange T, et al. [Patients' satisfaction after nasal septal and turbinate surgery. Results of a questionnaire]. Laryngorhinootologie 2007;86(10):706-713.

18. Gupta A, Mercurio E, Bielamowicz S. Endoscopic inferior turbinate reduction: An outcomes analysis. Laryngoscope 2001;111(11 Pt 1):1957-1959.

19. Hol MK, Huizing EH. Treatment of inferior turbinate pathology: A review and critical evaluation of the different techniques. Rhinology 2000;38(4):157-166.

20. Ho WK, Yuen AP, Tang KC, Wei WI, Lam PK. Time course in the relief of nasal blockage after septal and turbinate surgery: A prospective study. Arch Otolaryngol Head Neck Surg 2004;130(3):324-328.

21. Demirbas D, Cingi C, Cakli H, Kaya E. Use of rhinomanometry in common rhinologic disorders. Expert Rev Med Devices 2011;8(6):769-777. 